\title{
Describing readmissions to an Ebola case management centre (CMC), Sierra Leone, 2014
}

G Fitzpatrick (gabriel.fitzpatrick@gmail.com) ${ }^{1,2}$, F Vogt $^{3}$, O B Moi Gbabai ${ }^{4}$, B Black ${ }^{3}$, M Santantonio ${ }^{3}$, E Folkesson ${ }^{3}$, T Decroo5$^{5}$, M Van Herp ${ }^{3}$

1. Médecins Sans Frontières, Dublin, Ireland

2. Health Protection Surveillance Centre, Dublin, Ireland

3. Médecins Sans Frontières, Brussels, Belgium

4. Primary Health Care Unit Kailahun, Ministry of Health and Sanitation, Kailahun, Sierra Leone

5. Médecins Sans Frontières, Luxembourg

Citation style for this article:

Fitzpatrick G, Vogt F, Moi Gbabai OB, Black B, Santantonio M, Folkesson E, Decroo T, Van Herp M. Describing readmissions to an Ebola case management centre (CMC), Sierra Leone, 2014. Euro Surveill. 2014;19(40):pii=20924. Available online: http://www.eurosurveillance.org/ViewArticle.aspx?Articleld=20924

Article submitted on 25 September 2014 / published on 9 October 2014

Case management centres (CMCs) are part of the outbreak control plan for Ebola virus disease (EVD). A CMC in Sierra Leone had 33\% (138/419) of primary admissions discharged as EVD negative (not a case). Fifteen of these were readmitted within 21 days, nine of which were EVD positive. All readmissions had contact with an Ebola case in the community in the previous 21 days indicating that the infection was likely acquired outside the CMC.

Between 26 June and 1 September 2014, 138 patients were discharged from the Kailahun Ebola case management centre (CMC) in Sierra Leone, as non-Ebola virus disease (EVD) cases, because they tested negative for the virus by polymerase chain reaction (PCR). Of these, 15 returned to the CMC within 21 days of their first admission and subsequently nine tested positive for Ebola virus. This raised the question as to whether CMCs could be acting as potential amplifiers of infection even though appropriate infection control measures are being followed. Such a question is of public health importance to the overall future control of the EVD outbreak, which is ongoing in West Africa [1]. To our knowledge, there is no literature available which describes the evolution of readmissions to Ebola CMCs during an outbreak and this paper addresses that deficit.

\section{Ebola virus disease outbreak in West Africa} The current EVD outbreak in West Africa commenced in Guinea in December 2013 [1] and since then has spread to Sierra Leone, Liberia, Nigeria and Senegal [2]. It is the largest EVD outbreak recorded in history [2] with 6,553 (suspected, probable and confirmed) cases and 3,083 deaths reported as of 23 September 2014 in affected countries [2]. The World Health Organization (WHO) declared the outbreak a public health emergency of international concern on 8 August 2014 [3].

During EVD outbreaks transmission via infected body fluids occurs in three settings: (i) community, through contact with an infected person or contaminated fomites, (ii) burials, due to touching dead bodies, and (iii) nosocomial, via lack of infection control measures within healthcare facilities. In particular, the latter two settings [4] can quickly amplify an Ebola epidemic $[5,6]$. The incubation period of the virus ranges from two to 21 days $[5,7]$.

\section{Description on the Kailahun Ebola case management centre}

Médecins Sans Frontières (MSF) have six Ebola CMCs operational in West Africa, one of which is based in Kailahun, Sierra Leone. Suspected, probable and confirmed case definitions are equivalent to those used by the WHO [8]. In brief, a suspected case is any person, alive or dead, who has (or had) sudden onset of high fever and had contact with a person with suspected, probable or confirmed EVD or with a dead or sick animal; any person with sudden onset of high fever and at least three of the following symptoms: abdominal pain, anorexia, arthralgia, diarrhoea, dysphagia, dyspnoea, headache, hiccupping, lethargy, myalgia, or vomiting; or any person who had unexplained haemorrhagic symptoms or who died suddenly from an unexplained cause. A probable case is any person suspected to have EVD who was evaluated by a physician or any person who died from suspected EVD and had an epidemiological link with a confirmed case but was not tested and did not have laboratory confirmation of the disease. Suspect or probable cases are classified as confirmed when they had a positive laboratory test for EVD.

The Kailahun CMC (KCMC) is divided into a high risk zone and a low risk zone (Figure 1). The low risk zone includes the medical and nursing administrative tents, laundry area, storage area and other necessary facilities to support the high risk zone. Within the high risk zone personal protective equipment (PPE) must be worn at all times. The high risk zone comprises: a suspected cases ward, a probable cases ward and eight confirmed cases wards. A barrier fence separates the 
confirmed cases wards from the suspected and probable cases wards preventing patient interaction between these two types of wards.

Following medical assessment in triage, patients are referred to the suspected or probable cases ward depending on their case classification. An EVD PCR test (developed in-house by the Public Health Agency of Canada) on a blood sample is then performed. If this is positive the patient is transferred to the confirmed cases ward for further medical support while a negative result allows the patient to be discharged from the CMC. When a patient has a negative PCR result but symptom duration of less than 72 hours, a repeat PCR test is performed at 72 hours or more of symptoms to rule out a false negative result [9]. A patient can spend from less than 24 hours up to three days in the suspect/probable section of the CMC while awaiting the exclusion or confirmation of EVD. When PCR negative patients are discharged, they are considered exposed, and are added to the contact list. Patients who are discharged negative for EVD (not a case) from the suspect/ probable wards have the potential to be readmitted at a later date, and test either positive or negative for EVD. When readmissions test positive, they can cause anxiety among medical staff as they try to decipher if the patients have had any other EVD contact history apart from their previous primary assessment in the CMC.
Collection of readmission data at the Kailahun Ebola case management centre and data analyses

A patient register is maintained at the KCMC. It contains basic demographic, epidemiological, medical, laboratory and outcome data for each patient admitted to the facility in Excel 2010 format. All data are stored in a secure manner. To be classified as a readmission a patient must have at least two admission episodes to the CMC that have identical first name, surname, age, sex and address information. All patient readmissions since 26 June 2014 with their corresponding original admissions were extracted from the database. No time limit was imposed on the interval between admission and corresponding readmission when selecting cases. Outcomes for patients were classified as one of the following: cured, dead or not a case. Cured patients had been admitted with a positive EVD PCR and ultimately discharged alive with a negative EVD PCR. Patients classified as dead, had a positive EVD PCR at admission and subsequently died in the CMC from EVDrelated complications. The not a case outcome referred to patients who were admitted to the suspect or probable wards, tested negative for the virus by EVD PCR and were then discharged from the CMC.

The crude readmission ratio (CRR) was calculated as the total number of readmissions as a proportion of all 'not a case' primary discharges. Furthermore, the positive readmission ratio (PRR) was defined as the number

FIGURE 1

Outline map of Médecins Sans Frontières (MSF) Ebola case management centre (CMC)

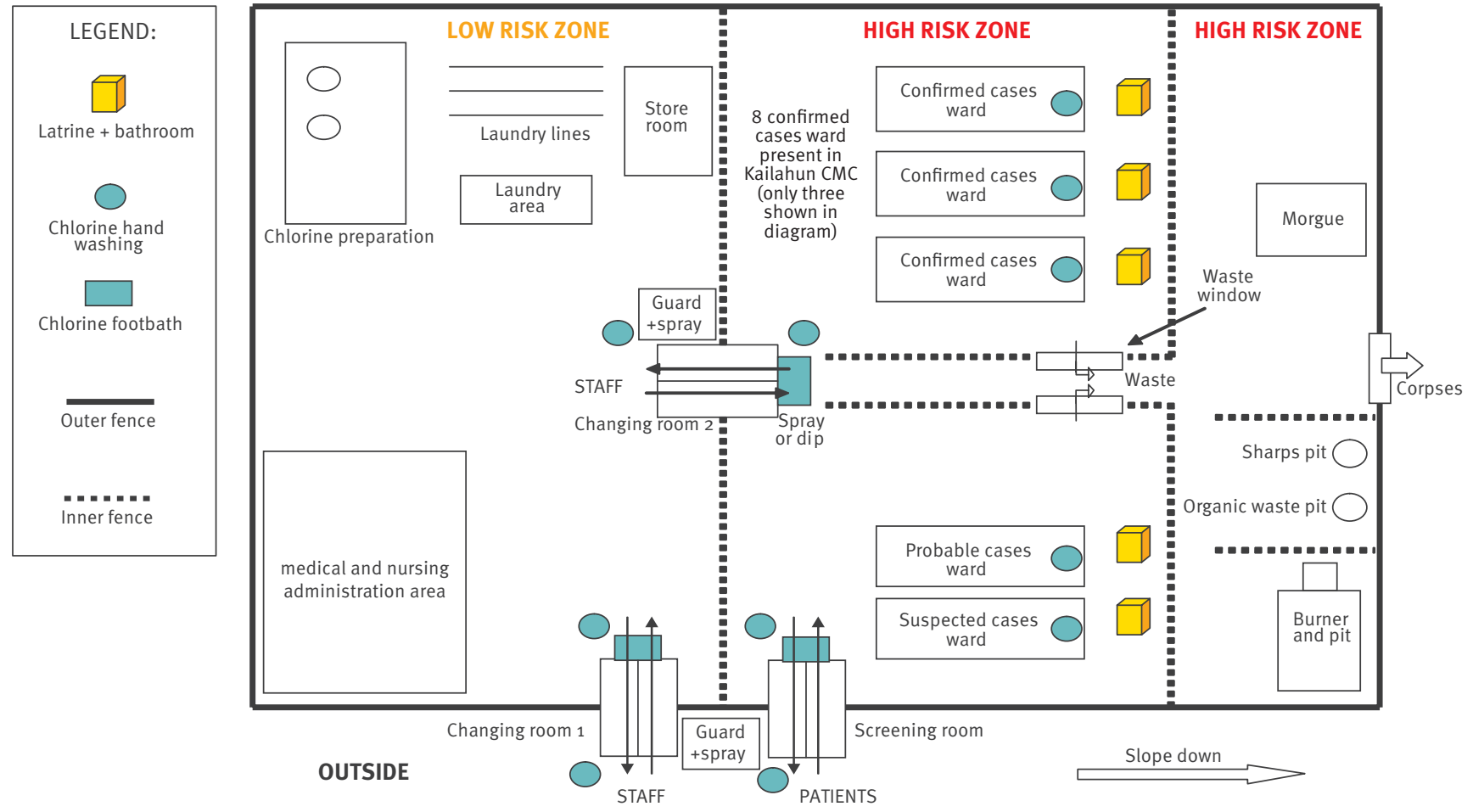

Source: Sterk E. Filovirus haemorrhagic fever guideline. Geneva: Médecins Sans Frontières; 2008. 
of readmissions with a positive EVD PCR as a proportion of all 'not a case' primary discharges.

This study fulfilled the MSF Ethics Review Board (Geneva, Switzerland) approved criteria for analysis of routinely collected anonymous programme data. All activities conducted by MSF were approved by the national authorities of Sierra Leone.

\section{Results}

Between 26 June and 1 September 2014 (study period), there were 419 primary admissions at the KCMC. Of these, 278 (66\%) were EVD PCR positive and 138 (33\%) were EVD PCR negative. Three ( $\$ 1 \%)$ admitted patients did not stay long enough in the centre to be tested for EVD (defaulters). During the same period there were 16 readmissions at KCMC. One readmission was

\section{FIGURE 2}

Distribution of readmissions to the Ebola case management centre (CMC), Kailahun, Sierra Leone, 26 June-1 September 2014 ( $\mathrm{n}=15$ readmissions)

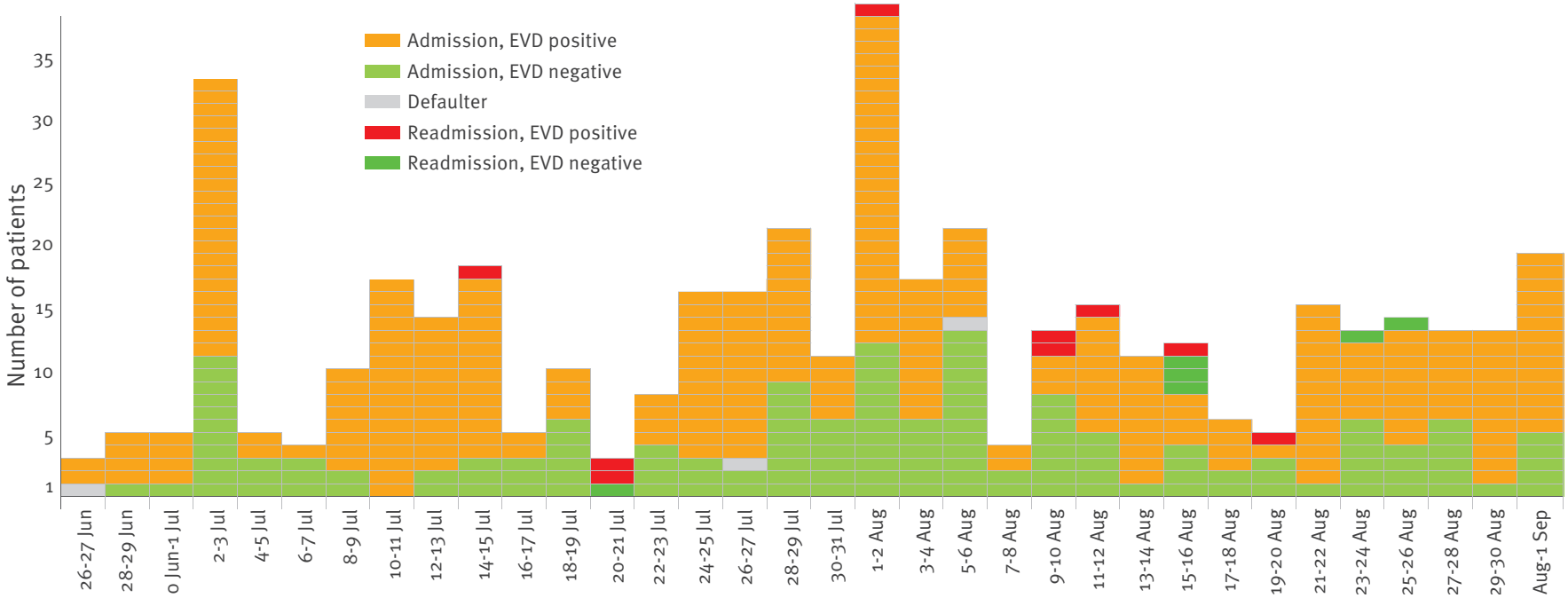

EVD: Ebola virus disease.

\section{TABLE 1}

Primary admission and corresponding readmission outcomes, Ebola case management centre (CMC), Kailahun, Sierra Leone, 26 June-1 September $2014(n=15)$

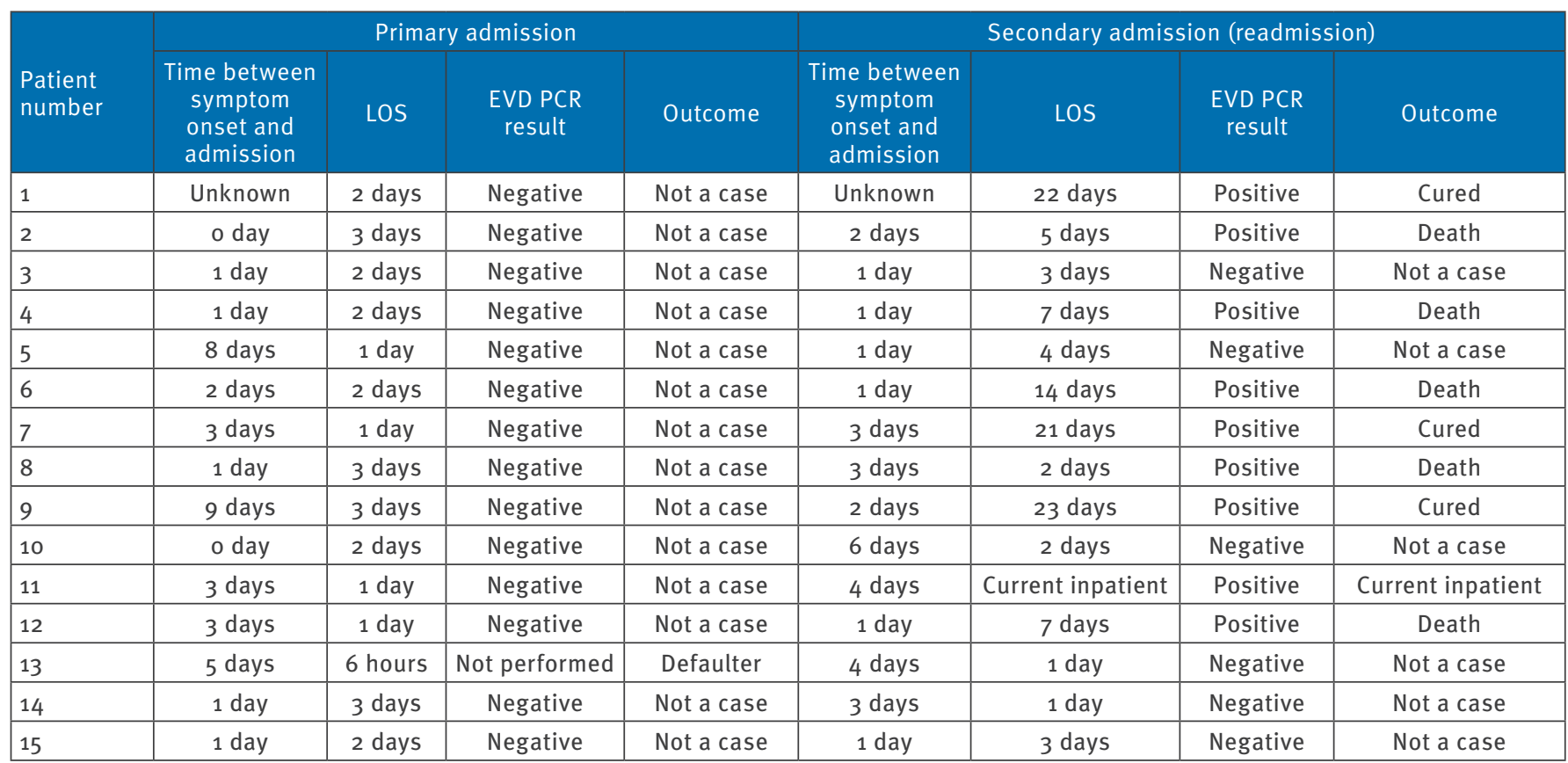

EVD: Ebola virus disease; LOS: length of stay; PCR: polymerase chain reaction. 
discordant for age (14 years versus 24 years) when compared with the original corresponding admission and was excluded from the analysis. The remaining 15 met the criteria to be defined as readmissions as described in the methodology. Taking these 15 readmissions into account, the KCMC had a total of 434 admissions during the study period, of which $239(55 \%)$ were male. The mean age of admissions was 29.9 years and 106 $(24 \%)$ were aged 18 years or less.

All 15 readmissions had only one previous admission. One patient did not have an EVD PCR result upon the first admission, as this person left the centre before testing could be done. The 14 remaining readmissions were all related to a prior admission whereby the PCR result was negative for EVD. The distribution of readmissions among all admissions to the KCMC is presented on the epidemiological curve in Figure2. It shows that four readmissions occurred during the first half of the outbreak while the remaining 11 presented in the second half.

Of the 15 readmissions, seven were male and four were aged 18 years or less. The mean age of readmissions was 27.9 years (range: $1.75-48$ years).

A positive EVD PCR test was obtained for nine readmissions of which five died, three were cured and one is a current inpatient at KCMC (Table 1). The crude readmission ratio (CRR) for KCMC was $11 \%(15 / 138)$ while the positive readmission ratio (PRR) was $7 \%(9 / 138)$. The average length of stay (LOS) at the KCMC for primary admissions linked to any readmission was 1.9 days (28/15) whereas the average LOS for primary admissions with corresponding EVD PCR positive and negative readmissions was $2(18 / 9)$ and 1.7 (10/6) days respectively. Regarding the three readmissions who were cured, they had an average LOS after readmission of 22 days (66/3) while the five readmissions who died and six who were not a case had an average LOS of seven (35/5) and $2.3(14 / 6)$ days respectively (Table 1$).$

The interval between discharge from primary admission and follow-up readmission to the KCMC for all readmissions was an average of 9.4 days with a range from four to 21 days (Table 2). Cases 1 to 15 also had a documented epidemiological contact with a suspected or confirmed case of Ebola (excluding their primary admission to the KCMC) within the prior 21 days to their readmission to the KCMC (Table 2). The majority (10/15) of these epidemiological contact types were household followed by occupational (3/15) and funeral (2/15) (Table 2).

\section{Discussion}

In response to the current EVD outbreak in West Africa, numerous Ebola CMCs are operating concurrently in the region [3]. MSF has previously set the standard for constructing and managing these centres in remote African settings $[9,10]$. The literature indicates that hospitals with inadequate infection control procedures have previously augmented filovirus outbreaks while appropriately run CMCs help contain them [4]. The emerging situation in Sierra Leone of patients who were initially discharged as non-cases from the KCMC and then returning as EVD PCR positive cases within 21 days has caused medical staff to question if CMCs are acting as potential amplifiers of infection during this outbreak even though appropriate infection control measures are being followed. Such a question is of public health importance to the overall future control of the outbreak.

This study has demonstrated that $7 \%$ of patients who were originally discharged as non-cases were readmitted as EVD PCR positive cases. Notably all readmissions occurred within 21 days of primary admission discharge, which is equivalent to the incubation period of EVD. This readmission's timeframe raises the possibility of nosocomial infection having occurred during the primary admission. The average LOS for primary admissions linked to positive readmissions was two days, during which time patients were admitted to the suspect and probable wards of the CMC. Infection control measures are strictly enforced in these wards, which are separated by barrier fencing from the confirmed wards in order to minimise the risk of nosocomial infection. Patients in the suspect/probable wards are encouraged to maintain a minimum distance from other patients at all times and not to touch or use items belonging to other patients. The number of cases per ward is capped to prevent overcrowding. Chlorine solution hand washing facilities are located at multiple points for patient and staff use. Patients can only be transferred from suspect/probable to confirmed wards and not vice versa to prevent spread of infection within the CMC. Hygienist staff regularly disinfects all areas within both the low and high risk zones. The implementation of strict infection control protocol in the suspect/ probable wards and the wider CMC in general reduces but can never eliminate the hazard of nosocomial EVD infection.

Importantly, all readmissions to the KCMC had documented epidemiological contacts with suspected or confirmed Ebola cases within the previous 21 days that did not include the original admission to the KCMC. This is a relatively reassuring finding as it acts as a counter weight to the fact that all readmissions occurred within the incubation period of EVD. The source of infection for positive readmissions is as likely to be the household, funeral and occupational contacts documented, as the primary admission to the KCMC. Positive readmissions partly reflect the continuous intense transmission of the virus in the surrounding community.

It is notable that patients who were discharged as not a case had an average LOS of almost two days in the suspect or probable wards. Unfortunately, it was not possible to distinguish between suspect and probable admissions and readmissions, as this information was not sufficiently recorded on the case investigation 


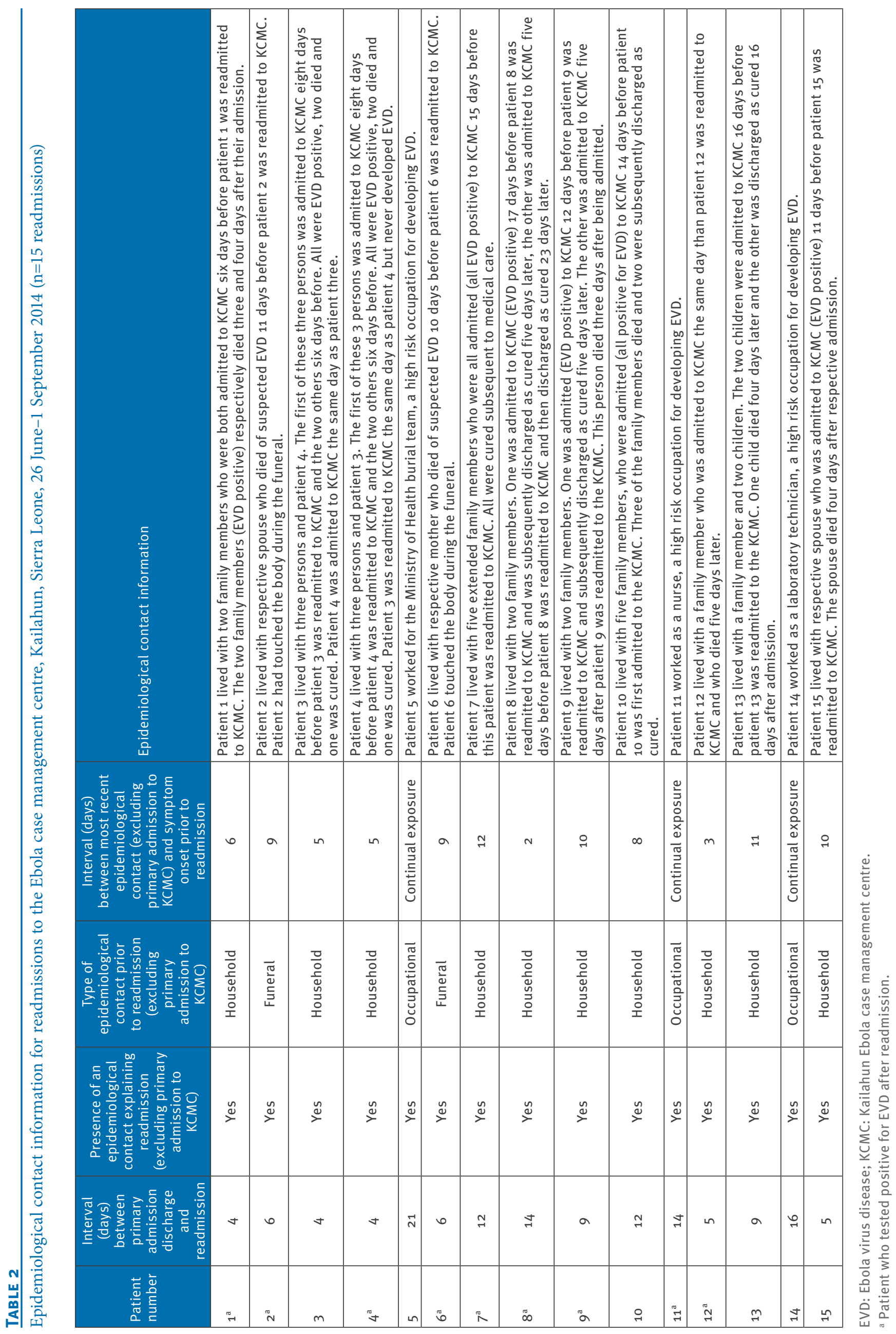


forms. Efforts are ongoing to collect this information in a more systematic manner in the field. There are multiple reasons for the LOS of almost two days including the lack of availability of a 24 hour laboratory service on-site to process blood samples and the restriction of the phlebotomy service to morning times only due to staff workload and safety concerns regarding performing venesection at night time. A proportion of newly admitted patients will require a repeat EVD PCR test if symptom duration has been less than 72 hours to rule out a false negative result [9]. In such cases the symptomatic patient will have to spend additional time in the suspect or probable ward until a repeat test is performed at the appropriate time. However, for newly arrived patients who already had a minimum of three days of symptoms, it is imperative that phlebotomy and laboratory analysis be performed as quickly as reasonably possible in order to prevent the risk of potential nosocomial EVD infection to patients who could be non-cases staying overnight in the suspect or probable wards. Ideally, phlebotomy and laboratory analysis at the CMC should be provided on a 24 hour basis where feasible. Furthermore, new bedside rapid diagnostic tests (RDT) for EVD that do not require phlebotomy are urgently needed. Such technology improves the timeliness of diagnosis for patients and reduces the risk of infections for healthcare staff.

The epidemiological curve showed that the majority of readmissions occurred during the second half of the outbreak to date. Readmissions can only develop from the pool of discharged non-cases because EVD positive cases have immunity to the specific strain if they survive to discharge $[11,12]$. On further inspection of the epidemiological curve it appears that positive readmissions have clustered following peaks in primary admissions. The clustering of three positive readmissions between 15 and 21 July and five positive readmissions between 10 and 19 August occurred within 21 days of the primary admissions peaks on 2 and 3 July and on 1 and 2 August respectively. The clustering of readmissions following primary admission peaks within the EVD incubation period suggests the possibility of the presence of superspreaders of the virus.

This study has shown the importance of analysing CMC readmissions to understand what exposures contribute to positive readmissions and to detect potential nosocomial EVD infection when no other sources of infection can be identified. For all positive readmissions described in this study an exposure, in addition to the primary admission, was identified within the EVD incubation period.

\section{Acknowledgements}

To our MSF friends and colleagues who have died while treating patients with Ebola in Sierra Leone. Their courage inspires us all.
Conflict of interest

None declared.

Author's contributions

Gabriel Fitzpatrick collected data in the MSF CMC in Sierra Leone and wrote the first draft of the paper and incorporated all co-authors comments into the final draft of the paper. Florian Vogt, Osman Bamba Moi Gbabai, Benjamin Black, Maud Santantonio, Elin Folkesson, Tom Decroo and Michel Van Herp all reviewed the paper and submitted their comments, which were included in the final draft of the paper.

\section{References}

1. Baize S, Pannetier D, Oestereich L, Rieger T, Koivogui L, Magassouba N, et al. Emergence of Zaire Ebola Virus Disease in Guinea. N Engl J Med. 2014;371(15):1418-25. http://dx.doi. org/10.1056/NEJMoa1404505

2. World Health Organization (WHO). Ebola response roadmap update. Geneva:WHO; Sep 2014. Available from: http://apps.who.int/iris/bitstream/10665/135029/1/ roadmapupdate26sept14_eng.pdf

3. World Health Organization (WHO). Ebola response roadmap. Geneva: WHO; Aug 2014. Available from: http://www.who.int/ csr/resources/publications/ebola/response-roadmap/en/

4. Ftika L, Maltezou HC. Viral haemorrhagic fevers in healthcare settings. J Hosp Infect. 2013;83(3):185-92. http://dx.doi. org/10.1016/j.jhin.2012.10.013

5. Feldmann H, Geisbert TW. Ebola haemorrhagic fever. Lancet. 2011;377(9768):849-62. http://dx.doi.org/10.1016/ S0140-6736(10)60667-8

6. MacNeil A, Rollin PE. Ebola and Marburg hemorrhagic fevers: neglected tropical diseases? PLoS Negl Trop Dis. 2012;6(6): e1546.

7. Dowell SF, Mukunu R, Ksiazek TG, Khan AS, Rollin PE, Peters CJ. Transmission of Ebola hemorrhagic fever: a study of risk factors in family members, Kikwit, Democratic Republic of the Congo, 1995. Commission de Lutte contre les Epidémies à Kikwit. J Infect Dis. 1999;179(Suppl 1):S87-91. http://dx.doi. org/10.1086/514284

8. World Health Organization (WHO). Case definition recommendations for Ebola or Marburg Virus Diseases. Geneva: WHO; Aug 2014. Available from: http://www.who. int/csr/resources/publications/ebola/ebola-case-definitioncontact-en.pdf?ua=1

9. Sterk E. Filovirus haemorrhagic fever guideline. Geneva: Médecins Sans Frontières; 2008.

10. Kerstiëns B, Matthys F. Interventions to control virus transmission during an outbreak of Ebola hemorrhagic fever: experience from Kikwit, Democratic Republic of the Congo, 1995. J Infect Dis. 1999;179(Suppl 1):S263-7. http://dx.doi. org/10.1086/514320

11. Wauquier N, Becquart P, Gasquet C, Leroy EM. Immunoglobulin $\mathrm{G}$ in Ebola outbreak survivors, Gabon. Emerg Infect Dis. 2009;15(7):1136-7. http://dx.doi.org/10.3201/eid1507.090402

12. Leroy EM, Gonzalez JP, Baize S. Ebola and Marburg haemorrhagic fever viruses: major scientific advances, but a relatively minor public health threat for Africa. Clin Microbiol Infect. 2011;17(7):964-76. http://dx.doi. org/10.1111/j.1469-0691.2011.03535.x 